Proceedings Book of International Work shop on Theory of Submanifolds (Volume: 1(2016)) June 2-4, 2016, Istanbul, Turkey. Editors: Nurettin Cenk Turgay, Elif Ozkara Canfes, Joeri Van der Veken and Cornelia-Livia Bejan Recieved: May 9, 2017 Accepted: July 17,2017

\title{
Rotational Surfaces with Pointwise 1-Type Gauss Map in Pseudo Euclidean Space $\mathbb{E}_{2}^{4}$
}

\author{
Ferdağ Kahraman Aksoyak, Yusuf Yaylı
}

Ferdağ Kahraman Aksoyak: Ahi Evran University, Division of Elementary Mathematics Education, Kirsehir, Turkey, e-mail:ferdag.aksoyak@ahievran.edu.tr,

Yusuf Yaylı: Ankara University, Department of Mathematics, Ankara, Turkey, email:yayli@science.ankara.edu.tr

\begin{abstract}
In this paper, we study rotational surfaces of elliptic, hyperbolic and parabolic type with pointwise 1-type Gauss map which have spacelike profile curve in four dimensional pseudo Euclidean space $\mathbb{E}_{2}^{4}$ and obtain some characterizations for these rotational surfaces to have pointwise 1-type Gauss map.

Keywords. Pseudo-Euclidean space - Rotational surfaces of elliptic, hyperbolic and parabolic type. Gauss map . Pointwise 1-type Gauss map.

MSC 2010 Classification. Primary: 53B25; Secondary:53C50.
\end{abstract}

\section{$1 \quad$ INTRODUCTION}

The Gauss map $G$ of a submanifold $M$ into $G(n, m)$ in $\wedge^{n} \mathbb{E}_{s}^{m}$, where $G(n, m)$ is the Grassmannian manifold consisting of all oriented $n$-planes through the origin of $\mathbb{E}_{s}^{m}$ and $\wedge^{n} \mathbb{E}_{s}^{m}$ is the vector space obtained by the exterior product of $n$ vectors in $\mathbb{E}_{s}^{m}$ is a smooth map which carries a point $p$ in $M$ into the oriented $n$-plane in $\mathbb{E}_{s}^{m}$ obtained from parallel translation of the tangent space of $M$ at $p$ in $\mathbb{E}_{s}^{m}$. Since the vector space $\wedge^{n} \mathbb{E}_{s}^{m}$ identify with a semi-Euclidean space $\mathbb{E}_{t}^{N}$ for some positive integer $t$, where $N=\left(\begin{array}{c}m \\ n\end{array}\right)$, the Gauss map is defined by $G: M \rightarrow G(n, m) \subset \mathbb{E}_{t}^{N}, G(p)=\left(e_{1} \wedge \ldots \wedge e_{n}\right)(p)$. The notion of submanifolds with finite type Gauss map was introduced by B. Y.Chen and P.Piccinni in 1987 [6] and after then many works were done about this topic, especially 1-type Gauss map and 2- type Gauss map.

If a submanifold $M$ of a Euclidean space or pseudo-Euclidean space has 1-type Gauss map $G$, then $G$ satisfies

$$
\Delta G=\lambda(G+C)
$$


for some $\lambda \in \mathbb{R}$ and some constant vector $C$.

On the other hand the Laplacian of the Gauss map of some typical wellknown surfaces satisfies the form

$$
\Delta G=f(G+C)
$$

for some smooth function $f$ on $M$ and some constant vector $C$. A submanifold of a Euclidean space or pseudo-Euclidean space is said to have pointwise 1-type Gauss map, if its Gauss map satisfies (1.1) for some smooth function $f$ on $M$ and some constant vector $C$. If the vector $C$ in (1.1) is zero, a submanifold with pointwise 1-type Gauss map is said to be of the first kind, otherwise it is said to be of the second kind.

A lot of papers were recently published about rotational surfaces with pointwise 1-type Gauss map in four dimensional Euclidean and pseudo Euclidean space in [1],[3],[4], [8], [9] [11].Timelike and spacelike rotational surfaces of elliptic, hyperbolic and parabolic types in Minkowski space $\mathbb{E}_{1}^{4}$ with pointwise 1-type Gauss map were studied in [5, 7]. Aksoyak and Yaylı in [2] studied boost invariant surfaces (rotational surfaces of hyperbolic type) with pointwise 1-type Gauss map in Minkowski space $\mathbb{E}_{1}^{4}$. They gave a characterization for flat boost invariant surfaces with pointwise 1-type Gauss map. Also they obtain some results for boost invariant marginally trapped surfaces with pointwise 1-type Gauss map. Ganchev and Milousheva in [10] defined three types of rotational surfaces with two dimensional axis rotational surfaces of elliptic, hyperbolic and parabolic type in pseudo Euclidean space $\mathbb{E}_{2}^{4}$. They classify all rotational marginally trapped surfaces of elliptic, hyperbolic and parabolic type, respectively.

In this paper, we study rotational surfaces of elliptic, hyperbolic and parabolic type with pointwise 1-type Gauss map which have spacelike profile curve in four dimensional pseudo Euclidean space and give all classifications of flat rotational

surfaces of elliptic, hyperbolic and parabolic type with pointwise 1-type Gauss map.

\section{$2 \quad$ PRELIMINARIES}

Let $\mathbb{E}_{s}^{m}$ be the $m$-dimensional pseudo-Euclidean space with signature $(s, m-s)$. Then the metric tensor $g$ in $\mathbb{E}_{s}^{m}$ has the form

$$
g=\sum_{i=1}^{m-s}\left(d x_{i}\right)^{2}-\sum_{i=m-s+1}^{m}\left(d x_{i}\right)^{2}
$$

where $\left(x_{1}, \ldots, x_{m}\right)$ is a standard rectangular coordinate system in $\mathbb{E}_{s}^{m}$.

A vector $v$ is called spacelike (resp., timelike) if $\langle v, v\rangle>0$ (resp., $\langle v, v\rangle<0$ ). Avector $v$ is called lightlike if it $v \neq 0$ and $\langle v, v\rangle=0$, where $\langle$,$\rangle is indefinite$ inner scalar product with respect to $g$. 
Let $M$ be an $n$-dimensional pseudo-Riemannian submanifold of a $m$-dimensional pseudo-Euclidean space $\mathbb{E}_{s}^{m}$ and denote by $\tilde{\nabla}$ and $\nabla$ Levi-Civita connections of $\mathbb{E}_{s}^{m}$ and $M$, respectively. We choose local orthonormal frame $\left\{e_{1}, \ldots, e_{n}, e_{n+1}, \ldots, e_{m}\right\}$ on $M$ with $\varepsilon_{A}=\left\langle e_{A}, e_{A}\right\rangle= \pm 1$ such that $e_{1}, \ldots e_{n}$ are tangent to $M$ and $e_{n+1}, \ldots, e_{m}$ are normal to $M$. We use the following convention on the ranges of indices: $1 \leq i, j, k, \ldots \leq n, n+1 \leq r, s, t, \ldots \leq m$, $1 \leq A, B, C, \ldots \leq m$.

Denote by $\omega_{A}$ the dual-1 form of $e_{A}$ such that $\omega_{A}(X)=\left\langle e_{A}, X\right\rangle$ and $\omega_{A B}$ the connection forms defined by

$$
d e_{A}=\sum_{B} \varepsilon_{B} \omega_{A B} e_{B}, \quad \omega_{A B}+\omega_{B A}=0 .
$$

Then the formulas of Gauss and Weingarten are given by

$$
\tilde{\nabla}_{e_{k}} e_{i}=\sum_{j=1}^{n} \varepsilon_{j} \omega_{i j}\left(e_{k}\right) e_{j}+\sum_{r=n+1}^{m} \varepsilon_{r} h_{i k}^{r} e_{r}
$$

and

$$
\tilde{\nabla}_{e_{k}} e_{s}=-\sum_{j=1}^{n} \varepsilon_{j} h_{k j}^{s} e_{j}+D_{e_{k}} e_{s}, \quad D_{e_{k}} e_{s}=\sum_{r=n+1}^{m} \varepsilon_{r} \omega_{s r}\left(e_{k}\right) e_{r},
$$

where $D$ is the normal connection, $h_{i k}^{r}$ the coefficients of the second fundamental form $h$.

For any real function $f$ on $M$, the Laplacian operator of $M$ with respect to induced metric is given by

$$
\Delta f=-\varepsilon_{i} \sum_{i}\left(\tilde{\nabla}_{e_{i}} \tilde{\nabla}_{e_{i}} f-\tilde{\nabla}_{\nabla_{e_{i}} e_{i}} f\right) .
$$

The mean curvature vector $H$ and the Gaussian curvature $K$ of $M$ in $\mathbb{E}_{s}^{m}$ are defined by

$$
H=\frac{1}{n} \sum_{s=n+1}^{m} \sum_{i=1}^{n} \varepsilon_{i} \varepsilon_{s} h_{i i}^{s} e_{s}
$$

and

$$
K=\sum_{s=n+1}^{m} \varepsilon_{s}\left(h_{11}^{s} h_{22}^{s}-h_{12}^{s} h_{21}^{s}\right),
$$

respectively. We recall that a surface $M$ is called minimal if its mean curvature vector vanishes identically, i.e. $H=0$. If the mean curvature vector satisfies $D H=0$, then the surface $M$ is said to have parallel mean curvature vector. Also if Gaussian curvature of $M$ vanishes identically, i.e. $K=0$, the surface $M$ is called flat. 
In this section, we consider rotational surfaces of elliptic, hyperbolic and parabolic type in four dimensional pseudo-Euclidean space $\mathbb{E}_{2}^{4}$ which are defined by Ganchev and Milousheva in [10] and investigate these rotational surfaces with pointwise 1-type Gauss map.

We denote the standart orthonormal basis of $\mathbb{E}_{2}^{4}$ by $\left\{\epsilon_{1}, \epsilon_{2}, \epsilon_{3}, \epsilon_{4}\right\}$ where $\epsilon_{1}=$ $(1,0,0,0), \epsilon_{2}=(0,1,0,0), \epsilon_{3}=(0,0,1,0)$ and $\epsilon_{4}=(0,0,0,1)$, and $\left\langle\epsilon_{1}, \epsilon_{1}\right\rangle=$ $\left\langle\epsilon_{2}, \epsilon_{2}\right\rangle=1,\left\langle\epsilon_{3}, \epsilon_{3}\right\rangle=\left\langle\epsilon_{4}, \epsilon_{4}\right\rangle=-1$.

\subsection{Rotational surfaces of elliptic type with pointwise 1-type Gauss map in $\mathbb{E}_{2}^{4}$}

In this subsection, first we consider the rotational surfaces of elliptic type with harmonic Gauss map.Then, we give a characterization of the flat rotational surfaces of elliptic type with pointwise 1-type Gauss map and obtain a relationship for non-minimal these surfaces with parallel mean curvature vector and pointwise 1-type Gauss map of the first kind.

Rotational surface of elliptic type $M_{1}$ is defined by

$$
\begin{gathered}
\varphi(t, s)=\left(\begin{array}{cccc}
1 & 0 & 0 & 0 \\
0 & 1 & 0 & 0 \\
0 & 0 & \cos t & -\sin t \\
0 & 0 & \sin t & \cos t
\end{array}\right)\left(\begin{array}{c}
x_{1}(s) \\
x_{2}(s) \\
x_{3}(s) \\
0
\end{array}\right) \\
M_{1}: \varphi(t, s)=\left(x_{1}(s), x_{2}(s), x_{3}(s) \cos t, x_{3}(s) \sin t\right),
\end{gathered}
$$

where the surface $M_{1}$ is obtained by the rotation of the curve

$$
x(s)=\left(x_{1}(s), x_{2}(s), x_{3}(s), 0\right)
$$

about the two dimensional Euclidean plane $\operatorname{span}\left\{\epsilon_{1}, \epsilon_{2}\right\}$. Let the profile curve of $M_{1}$ be unit speed spacelike curve. In that case, $\left(x_{1}{ }^{\prime}(s)\right)^{2}+\left(x_{2}{ }^{\prime}(s)\right)^{2}-\left(x_{3}{ }^{\prime}(s)\right)^{2}=$ 1. We suppose that $x_{3}(s)>0$. The moving frame field $\left\{e_{1}, e_{2}, e_{3}, e_{4}\right\}$ on $M_{1}$ is determined as follows:

$$
\begin{aligned}
e_{1}= & \left(x_{1}^{\prime}(s), x_{2}^{\prime}(s), x_{3}^{\prime}(s) \cos t, x_{3}^{\prime}(s) \sin t\right) \\
e_{2}= & (0,0,-\sin t, \cos t) \\
e_{3}= & \frac{1}{\sqrt{1+x_{3}^{\prime}(s)^{2}}}\left(-x_{2}^{\prime}(s), x_{1}^{\prime}(s), 0,0\right) \\
e_{4}= & \frac{1}{\sqrt{1+x_{3}^{\prime}(s)^{2}}}\left(x_{3}^{\prime}(s) x_{1}^{\prime}(s), x_{3}^{\prime}(s) x_{2}^{\prime}(s),\left(1+x_{3}^{\prime}(s)^{2}\right) \cos t\right. \\
& \left.\left(1+x_{3}^{\prime}(s)^{2}\right) \sin t\right)
\end{aligned}
$$


where $e_{1}, e_{2}$ and $e_{3}, e_{4}$ are tangent vector fields and normal vector fields to $M_{1}$, respectively. Then it is easily seen that

$$
\left\langle e_{1}, e_{1}\right\rangle=\left\langle e_{3}, e_{3}\right\rangle=1,\left\langle e_{2}, e_{2}\right\rangle=\left\langle e_{4}, e_{4}\right\rangle=-1 .
$$

We have the dual 1-forms as:

$$
\omega_{1}=d s \quad \text { and } \quad \omega_{2}=-x_{3}(s) d t .
$$

After some computations, the components of the second fundamental form and the connection forms are given as follows:

$$
\begin{aligned}
& h_{11}^{3}=-d(s), \quad h_{12}^{3}=0, \quad h_{22}^{3}=0, \\
& h_{11}^{4}=-c(s), \quad h_{12}^{4}=0, \quad h_{22}^{4}=b(s)
\end{aligned}
$$

and

$$
\begin{aligned}
& \omega_{12}=a(s) b(s) \omega_{2}, \quad \omega_{13}=-d(s) \omega_{1}, \quad \omega_{14}=-c(s) \omega_{1}, \\
& \omega_{23}=0, \quad \omega_{24}=-b(s) \omega_{2}, \quad \omega_{34}=a(s) d(s) \omega_{1} .
\end{aligned}
$$

By taking the covariant derivative with respect to $e_{1}$ and $e_{2}$ we have

$$
\begin{aligned}
\tilde{\nabla}_{e_{1}} e_{1} & =-d(s) e_{3}+c(s) e_{4}, \\
\tilde{\nabla}_{e_{2}} e_{1} & =a(s) b(s) e_{2}, \\
\tilde{\nabla}_{e_{1}} e_{2} & =0, \\
\tilde{\nabla}_{e_{2}} e_{2} & =a(s) b(s) e_{1}-b(s) e_{4}, \\
\tilde{\nabla}_{e_{1}} e_{3} & =d(s) e_{1}-a(s) d(s) e_{4}, \\
\tilde{\nabla}_{e_{2}} e_{3} & =0, \\
\tilde{\nabla}_{e_{1}} e_{4} & =c(s) e_{1}-a(s) d(s) e_{3}, \\
\tilde{\nabla}_{e_{2}} e_{4} & =b(s) e_{2},
\end{aligned}
$$

where

$$
\begin{aligned}
a(s) & =\frac{x_{3}^{\prime}(s)}{\sqrt{1+\left(x_{3}^{\prime}\right)^{2}}}, \\
b(s) & =\frac{\sqrt{1+\left(x_{3}^{\prime}\right)^{2}}}{x_{3}(s)}, \\
c(s) & =\frac{x_{3}^{\prime \prime}(s)}{\sqrt{1+\left(x_{3}^{\prime}\right)^{2}}}, \\
d(s) & =\frac{x_{1}^{\prime \prime}(s) x_{2}^{\prime}(s)-x_{2}^{\prime \prime}(s) x_{1}^{\prime}(s)}{\sqrt{1+\left(x_{3}^{\prime}\right)^{2}}} .
\end{aligned}
$$

By using (2.2), (2.3) and (3.2), the mean curvature vector and Gaussian curvature of the surface $M_{1}$ are obtained as:

$$
H=\frac{1}{2}\left(-d(s) e_{3}+(c(s)+b(s)) e_{4}\right)
$$


and

$$
K=c(s) b(s)
$$

respectively.

By using (2.1) and (3.3), we find the Laplacian of the Gauss map of $M_{1}$ as :

$$
\Delta G=L(s)\left(e_{1} \wedge e_{2}\right)+M(s)\left(e_{2} \wedge e_{3}\right)+N(s)\left(e_{2} \wedge e_{4}\right),
$$

where

$$
\begin{gathered}
L(s)=d^{2}(s)-b^{2}(s)-c^{2}(s), \\
M(s)=d^{\prime}(s)+a(s) d(s)(b(s)+c(s)), \\
N(s)=b^{\prime}(s)+c^{\prime}(s)+a(s) d^{2}(s) .
\end{gathered}
$$

Theorem 3.1. Let $M_{1}$ be rotation surface of elliptic type given by the parametrization (3.1). If $M_{1}$ has harmonic Gauss map then it has constant Gaussian curvature.

Proof. Let the Gauss map of $M_{1}$ be harmonic, i.e., $\Delta G=0$. So, from (3.10), (3.11), (3.12) and (3.13) we have

$$
\begin{aligned}
d^{2}(s)-b^{2}(s)-c^{2}(s) & =0, \\
d^{\prime}(s)+a(s) d(s)(b(s)+c(s)) & =0 \\
b^{\prime}(s)+c^{\prime}(s)+a(s) d^{2}(s) & =0 .
\end{aligned}
$$

By multiplying both sides of the second equation of (3.14) with $d(s)$ and using the third equation of (3.14) we have

$$
d(s) d^{\prime}(s)-b(s) b^{\prime}(s)-c(s) c^{\prime}(s)=(b(s) c(s))^{\prime} .
$$

By differeniating the first equation of (3.14) with respect to $s$ and using (3.15), we have that $b(s) c(s)=$ constant.Hence, from (3.9) we get $K=$ $K_{0}=$ constant.

Theorem 3.2. Let $M_{1}$ be the flat rotational surface of elliptic type given by the parametrization (3.1). Then $M_{1}$ has a pointwise 1-type Gauss map if and only if the profile curve of $M_{1}$ is characterized by one of the following way:

i)

$$
\begin{aligned}
& x_{1}(s)=-\frac{1}{\delta_{1}} \sin \left(-\delta_{1} s+\delta_{2}\right)+\delta_{4}, \\
& x_{2}(s)=\frac{1}{\delta_{1}} \cos \left(-\delta_{1} s+\delta_{2}\right)+\delta_{4}, \\
& x_{3}(s)=\delta_{3},
\end{aligned}
$$

where $\delta_{1}, \delta_{2}, \delta_{3}$ and $\delta_{4}$ are real constants and the Gauss map of $M_{1}$ satisfies (1.1) for $f=\delta_{1}^{2}-\frac{1}{\delta_{3}^{2}}$ and $C=0$. If $\delta_{1} \delta_{3}= \pm 1$ then the function $f$ becomes zero and it implies that the Gauss map is harmonic. 
ii)

$$
\begin{aligned}
& x_{1}(s)=\int\left(1+\lambda_{1}^{2}\right)^{\frac{1}{2}} \cos \left(-\frac{\lambda_{3}}{\lambda_{1}\left(1+\lambda_{1}^{2}\right)^{\frac{1}{2}}} \ln \left(\lambda_{1} s+\lambda_{2}\right)+\lambda_{4}\right) d s, \\
& x_{2}(s)=\int\left(1+\lambda_{1}^{2}\right)^{\frac{1}{2}} \sin \left(-\frac{\lambda_{3}}{\lambda_{1}\left(1+\lambda_{1}^{2}\right)^{\frac{1}{2}}} \ln \left(\lambda_{1} s+\lambda_{2}\right)+\lambda_{4}\right) d s, \\
& x_{3}(s)=\lambda_{1} s+\lambda_{2},
\end{aligned}
$$

where $\lambda_{1}, \lambda_{2}, \lambda_{3}$ and $\lambda_{4}$ are real constants and the Gauss map of $M_{1}$ satisfies (1.1) for $f(s)=\frac{1}{\left(\lambda_{1} s+\lambda_{2}\right)^{2}}\left(\frac{\lambda_{3}^{2}}{1+\lambda_{1}^{2}}-1\right)$ and $C=\lambda_{1}^{2} e_{1} \wedge e_{2}+\lambda_{1}\left(1+\lambda_{1}^{2}\right)^{\frac{1}{2}} e_{2} \wedge e_{4}$.

Proof. We suppose that $M_{1}$ has pointwise 1-type Gauss map. By using (1.1) and (3.10), we get

$$
\begin{aligned}
-f+f\left\langle C, e_{1} \wedge e_{2}\right\rangle & =-L(s), \\
f\left\langle C, e_{2} \wedge e_{3}\right\rangle & =-M(s), \\
f\left\langle C, e_{2} \wedge e_{4}\right\rangle & =N(s)
\end{aligned}
$$

and

$$
\left\langle C, e_{1} \wedge e_{3}\right\rangle=\left\langle C, e_{1} \wedge e_{4}\right\rangle=\left\langle C, e_{3} \wedge e_{4}\right\rangle=0
$$

By taking the derivatives of all equations in (3.19) with respect to $e_{2}$ and using (3.18) we obtain

$$
\begin{aligned}
a(s) N(s)-L(s)+f & =0, \\
a(s) M(s) & =0 \\
M(s) & =0,
\end{aligned}
$$

respectively. From above equations, we have two cases. One of them is $a(s)=0$, $M(s)=0$ and the other is $a(s) \neq 0, M(s)=0$. Firstly, we suppose that $a(s)=0$ and $M(s)=0$. By using (3.4), we have that $x_{3}(s)=\delta_{3}=$ constant. It implies that $c(s)=0, b(s)=\frac{1}{\delta_{3}}$ and $M_{1}$ is flat. Since the profile curve $x$ is spacelike curve which is parameterized by arc-length, we can put

$$
\begin{aligned}
& x_{1}^{\prime}(s)=\cos \delta(s)(\text { or resp. } \sin \delta(s)), \\
& \left.x_{2}^{\prime}(s)=\sin \delta(s) \text { (or resp. } \cos \delta(s)\right),
\end{aligned}
$$

where $\delta$ is smooth angle function. Without loss of generality we assume that

$$
x_{1}^{\prime}(s)=\cos \delta(s) \text { and } x_{2}^{\prime}(s)=\sin \delta(s)
$$

We can do similar computations for the another case, too. By using third equation of (3.20) and (3.12) we obtain that

$$
d(s)=\delta_{1}, \delta_{1} \text { is non zero constant. }
$$


On the other hand by using (3.7), (3.21) and (3.22) we get

$$
\delta(s)=-\delta_{1} s+\delta_{2},
$$

where $\delta_{1}, \delta_{2}$ are real constants. Then by substituting (3.23) into (3.21) and taking the integral we have the equation (3.16). Also the Laplacian of the Gauss map of $M_{1}$ with the equations $a(s)=0, b(s)=\frac{1}{\delta_{3}}, c(s)=0$ and $d(s)=\delta_{1}$ is found as $\Delta G=\left(\delta_{1}^{2}-\frac{1}{\delta_{3}^{2}}\right) G$

Now we suppose that $a(s) \neq 0$ and $M(s)=0$. Since the surface $M_{1}$ is flat, i.e., $K=0$. By using (3.9) we have that $c(s)=0$. From (3.6) we get

$$
x_{3}(s)=\lambda_{1} s+\lambda_{2}
$$

for some constants $\lambda_{1} \neq 0$ and $\lambda_{2}$. In that case by using (3.4), (3.5) and (3.24) we have

$$
a(s)=\frac{\lambda_{1}}{\left(1+\lambda_{1}^{2}\right)^{\frac{1}{2}}}
$$

and

$$
b(s)=\frac{\left(1+\lambda_{1}^{2}\right)^{\frac{1}{2}}}{\lambda_{1} s+\lambda_{2}} .
$$

Let consider that $M(s)=0$ with $c(s)=0$. In that case from (3.12), we obtain that

$$
d^{\prime}(s)+a(s) b(s) d(s)=0
$$

By using (3.25), (3.26) and (3.27) we have

$$
d(s)=\frac{\lambda_{3}}{\lambda_{1} s+\lambda_{2}},
$$

where $\lambda_{3}$ is constant of integration. On the other hand, Since the profile curve $x$ is spacelike curve which is parameterized by arc-length, we can put

$$
\begin{aligned}
& x_{1}^{\prime}(s)=\left(1+\lambda_{1}^{2}\right)^{\frac{1}{2}} \cos \lambda(s), \\
& x_{2}^{\prime}(s)=\left(1+\lambda_{1}^{2}\right)^{\frac{1}{2}} \sin \lambda(s),
\end{aligned}
$$

where $\lambda$ is smooth angle function. By differentiating (3.29). we obtain

$$
\begin{aligned}
& x_{1}^{\prime \prime}(s)=-\left(1+\lambda_{1}^{2}\right)^{\frac{1}{2}} \sin \lambda(s) \lambda^{\prime}(s), \\
& x_{2}^{\prime \prime}(s)=\left(1+\lambda_{1}^{2}\right)^{\frac{1}{2}} \cos \lambda(s) \lambda^{\prime}(s) .
\end{aligned}
$$

By using (3.7), (3.24), (3.29) and (3.30), we get

$$
d(s)=-\left(1+\lambda_{1}^{2}\right)^{\frac{1}{2}} \lambda^{\prime}(s) .
$$

By combining (3.28) and (3.31) we obtain

$$
\lambda(s)=-\frac{\lambda_{3}}{\lambda_{1}\left(1+\lambda_{1}^{2}\right)^{\frac{1}{2}}} \ln \left(\lambda_{1} s+\lambda_{2}\right)+\lambda_{4} .
$$


So by substituting (3.32) into (3.29), we get

$$
\begin{aligned}
& x_{1}(s)=\int\left(1+\lambda_{1}^{2}\right)^{\frac{1}{2}} \cos \left(-\frac{\lambda_{3}}{\lambda_{1}\left(1+\lambda_{1}^{2}\right)^{\frac{1}{2}}} \ln \left(\lambda_{1} s+\lambda_{2}\right)+\lambda_{4}\right) d s, \\
& x_{2}(s)=\int\left(1+\lambda_{1}^{2}\right)^{\frac{1}{2}} \sin \left(-\frac{\lambda_{3}}{\lambda_{1}\left(1+\lambda_{1}^{2}\right)^{\frac{1}{2}}} \ln \left(\lambda_{1} s+\lambda_{2}\right)+\lambda_{4}\right) d s,
\end{aligned}
$$

Conversely, the surface $M_{1}$ whose the profil curve given by (3.17) is pointwise 1-type Gauss map for

$$
f(s)=\frac{1}{\left(\lambda_{1} s+\lambda_{2}\right)^{2}}\left(\frac{\lambda_{3}^{2}}{1+\lambda_{1}^{2}}-1\right)
$$

and

$$
C=\lambda_{1}^{2} e_{1} \wedge e_{2}+\lambda_{1}\left(1+\lambda_{1}^{2}\right)^{\frac{1}{2}} e_{2} \wedge e_{4}
$$

Theorem 3.3. A non- minimal rotational surfaces of elliptic type $M_{1}$ defined by (3.1) has pointwise 1-type Gauss map of the first kind if and only if the mean curvature vector of $M_{1}$ is parallel.

Proof. From (3.8) we have that $H=\frac{1}{2}\left(-d(s) e_{3}+(c(s)+b(s)) e_{4}\right)$. Let the mean curvature vector of $M_{1}$ be parallel, i.e., $D H=0$. Then we get

$$
D_{e_{1}} H=\frac{1}{2}\left(-M(s) e_{3}+N(s) e_{4}\right)=0 .
$$

In this case we obtain that $M(s)=N(s)=0$. From (3.10), we have that $\Delta G=L(s) e_{1} \wedge e_{2}$.

Conversely, if $M_{1}$ has pointwise 1-type Gauss map of the first kind then from (3.10) we get $M(s)=N(s)=0$ and it implies that $M_{1}$ has parallel mean curvature vector.

Corollary 3.4. If rotational surfaces of elliptic type $M_{1}$ given by (3.1) is minimal then it has pointwise 1-type Gauss map of the first kind.

\subsection{Rotational surfaces of hyperbolic type with pointwise 1-type Gauss map in $\mathbb{E}_{2}^{4}$}

In this subsection, first we consider rotational surfaces of hyperbolic type with harmonic Gauss map. Moreover, we obtain a characterization of flat rotational surfaces of hyperbolic type with pointwise 1-type Gauss map and give a relationship for non-minimal these surfaces with parallel mean curvature vector and pointwise 1-type Gauss map of the first kind. The proofs of theorems in this subsection are similar the proofs of theorems in previous section so we give the theorems as without proof. 
Rotational surface of hyperbolic type $M_{2}$ is defined by

$$
\begin{gathered}
\varphi(t, s)=\left(\begin{array}{cccc}
\cosh t & 0 & \sinh t & 0 \\
0 & 1 & 0 & 0 \\
\sinh t & 0 & \cosh t & 0 \\
0 & 0 & 0 & 1
\end{array}\right)\left(\begin{array}{c}
x_{1}(s) \\
x_{2}(s) \\
0 \\
x_{4}(s)
\end{array}\right) \\
M_{2}: \varphi(t, s)=\left(x_{1}(s) \cosh t, x_{2}(s), x_{1}(s) \sinh t, x_{4}(s)\right),
\end{gathered}
$$

where the surface $M_{2}$ is obtained by the rotation of the curve

$$
x(s)=\left(x_{1}(s), x_{2}(s), 0, x_{4}(s)\right)
$$

about the two dimensional Euclidean plane spanned by $\epsilon_{2}$ and $\epsilon_{4}$. Let the profile curve of $M_{2}$ be unit speed spacelike curve. In that case $\left(x_{1}{ }^{\prime}(s)\right)^{2}+\left(x_{2}{ }^{\prime}(s)\right)^{2}-$ $\left(x_{4}{ }^{\prime}(s)\right)^{2}=1$. We assume that $x_{1}(s)>0$. The moving frame field $\left\{e_{1}, e_{2}, e_{3}, e_{4}\right\}$ on $M_{2}$ is choosen as follows:

$$
\begin{aligned}
e_{1}= & \left(x_{1}^{\prime}(s) \cosh t, x_{2}^{\prime}(s), x_{1}^{\prime}(s) \sinh t, x_{4}^{\prime}(s)\right) \\
e_{2}= & (\sinh t, 0, \cosh t, 0) \\
e_{3}= & \frac{1}{\sqrt{\varepsilon\left(x_{1}^{\prime}(s)^{2}-1\right)}}\left(0, x_{4}^{\prime}(s), 0, x_{2}^{\prime}(s)\right) \\
e_{4}= & \frac{1}{\sqrt{\varepsilon\left(x_{1}^{\prime}(s)^{2}-1\right)}}\left(\left(x_{1}^{\prime}(s)^{2}-1\right) \cosh t,-x_{1}^{\prime}(s) x_{2}^{\prime}(s),\left(x_{1}^{\prime}(s)^{2}-1\right) \sinh t\right. \\
& \left.-x_{1}^{\prime}(s) x_{4}^{\prime}(s)\right)
\end{aligned}
$$

where $e_{1}, e_{2}$ and $e_{3}, e_{4}$ are tangent vector fields and normal vector fields to $M_{2}$, respectively and $\varepsilon$ is signature of $\left(x_{1}{ }^{\prime}\right)^{2}-1$. If $\left(x_{1}{ }^{\prime}\right)^{2}-1$ is positive (resp. negative) then $\varepsilon=1$ (resp. $\varepsilon=-1$ ). It is easily seen that

$$
\left\langle e_{1}, e_{1}\right\rangle=-\left\langle e_{2}, e_{2}\right\rangle=1,\left\langle e_{3}, e_{3}\right\rangle=-\left\langle e_{4}, e_{4}\right\rangle=\varepsilon .
$$

we have the dual 1-forms as:

$$
\omega_{1}=d s \quad \text { and } \quad \omega_{2}=-x_{1}(s) d t .
$$

After some computations, components of the second fundamental form and the connection forms are obtained by:

$$
\begin{aligned}
& h_{11}^{3}=d(s), \quad h_{12}^{3}=0, \quad h_{22}^{3}=0, \\
& h_{11}^{4}=c(s), \quad h_{12}^{4}=0, \quad h_{22}^{4}=-\varepsilon b(s)
\end{aligned}
$$

and

$$
\begin{aligned}
& \omega_{12}=a(s) b(s) \omega_{2}, \quad \omega_{13}=d(s) \omega_{1}, \quad \omega_{14}=c(s) \omega_{1}, \\
& \omega_{23}=0, \quad \omega_{24}=\varepsilon b(s) \omega_{2}, \quad \omega_{34}=a(s) d(s) \omega_{1} .
\end{aligned}
$$


Differentiating covariantly with respect to $e_{1}$ and $e_{2}$ we get

$$
\begin{aligned}
\tilde{\nabla}_{e_{1}} e_{1} & =\varepsilon d(s) e_{3}-\varepsilon c(s) e_{4} \\
\tilde{\nabla}_{e_{2}} e_{1} & =a(s) b(s) e_{2} \\
\tilde{\nabla}_{e_{1}} e_{2} & =0 \\
\tilde{\nabla}_{e_{2}} e_{2} & =a(s) b(s) e_{1}+b(s) e_{4} \\
\tilde{\nabla}_{e_{1}} e_{3} & =-d(s) e_{1}-\varepsilon a(s) d(s) e_{4} \\
\tilde{\nabla}_{e_{2}} e_{3} & =0 \\
\tilde{\nabla}_{e_{1}} e_{4} & =-c(s) e_{1}-\varepsilon a(s) d(s) e_{3} \\
\tilde{\nabla}_{e_{2}} e_{4} & =-\varepsilon b(s) e_{2}
\end{aligned}
$$

where

$$
\begin{gathered}
a(s)=\frac{x_{1}^{\prime}(s)}{\sqrt{\varepsilon\left(\left(x_{1}^{\prime}\right)^{2}-1\right)}}, \\
b(s)=\frac{\sqrt{\varepsilon\left(\left(x_{1}^{\prime}\right)^{2}-1\right)}}{x_{1}(s)}, \\
c(s)=\frac{x_{1}^{\prime \prime}(s)}{\sqrt{\varepsilon\left(\left(x_{1}^{\prime}\right)^{2}-1\right)}}, \\
d(s)=\frac{x_{2}^{\prime \prime}(s) x_{4}^{\prime}(s)-x_{4}^{\prime \prime}(s) x_{2}^{\prime}(s)}{\sqrt{\varepsilon\left(\left(x_{1}^{\prime}\right)^{2}-1\right)}} .
\end{gathered}
$$

By using (2.2), (2.3) and (3.34), the mean curvature vector and Gaussian curvature of the surface $M_{2}$ are obtained as follows:

$$
H=\frac{1}{2}\left(\varepsilon d(s) e_{3}-\varepsilon(c(s)+\varepsilon b(s)) e_{4}\right)
$$

and

$$
K=c(s) b(s)
$$

respectively.

By using (2.1) and (3.35), we find the Laplacian of the Gauss map of $M_{2}$ as:

$$
\Delta G=L(s)\left(e_{1} \wedge e_{2}\right)+M(s)\left(e_{2} \wedge e_{3}\right)+N(s)\left(e_{2} \wedge e_{4}\right),
$$

where

$$
\begin{gathered}
L(s)=\varepsilon\left(d^{2}(s)-c^{2}(s)-b^{2}(s)\right), \\
M(s)=\varepsilon\left(d^{\prime}(s)+\varepsilon a(s) d(s)(c(s)+\varepsilon b(s))\right), \\
N(s)=-\varepsilon\left(c^{\prime}(s)+\varepsilon b^{\prime}(s)+\varepsilon a(s) d^{2}(s)\right) .
\end{gathered}
$$


Theorem 3.5. Let $M_{2}$ be rotation surface of hyperbolic type given by the parameterization (3.33). If $M_{2}$ has Gauss map harmonic then it has constant Gaussian curvatrure.

Theorem 3.6. Let $M_{2}$ be flat rotation surface of hyperbolic type given by the parameterization (3.33). Then $M_{2}$ has pointwise 1-type Gauss map if and only if the profile curve of $M_{2}$ is characterized in one of the following way:

i)

$$
\begin{aligned}
& x_{1}(s)=\delta_{1}, \\
& x_{2}(s)=-\frac{1}{\delta_{2}} \sinh \left(-\delta_{2} s+\delta_{3}\right)+\delta_{4}, \\
& x_{4}(s)=-\frac{1}{\delta_{2}} \cosh \left(-\delta_{2} s+\delta_{3}\right)+\delta_{4},
\end{aligned}
$$

where $\delta_{1}, \delta_{2}, \delta_{3}$ and $\delta_{4}$ are real constants and the Gauss map $G$ satisfies (1.1) for $f=\frac{1}{\delta_{1}^{2}}-\delta_{2}^{2}$ and $C=0$. If $\delta_{1} \delta_{2}= \pm 1$ then the function $f$ becomes zero and it implies that the Gauss map is harmonic.

ii)

$$
\begin{aligned}
& x_{1}(s)=\lambda_{1} s+\lambda_{2}, \\
& x_{2}(s)=\int\left(\lambda_{1}^{2}-1\right)^{\frac{1}{2}} \sinh \left(\frac{\lambda_{3}}{\lambda_{1}\left(\lambda_{1}^{2}-1\right)^{\frac{1}{2}}} \ln \left(\lambda_{1} s+\lambda_{2}\right)+\lambda_{4}\right) d s, \\
& x_{4}(s)=\int\left(\lambda_{1}^{2}-1\right)^{\frac{1}{2}} \cosh \left(\frac{\lambda_{3}}{\lambda_{1}\left(\lambda_{1}^{2}-1\right)^{\frac{1}{2}}} \ln \left(\lambda_{1} s+\lambda_{2}\right)+\lambda_{4}\right) d s,
\end{aligned}
$$

where $\lambda_{1}, \lambda_{2}, \lambda_{3}$ and $\lambda_{4}$ are real constants and without loss of generality we suppose that $\lambda_{1}^{2}-1>0$. Morever the Gauss map $G$ satisfies (1.1) for the function $f(s)=\frac{1}{\left(\lambda_{1} s+\lambda_{2}\right)^{2}}\left(1-\frac{\lambda_{3}^{2}}{\lambda_{1}^{2}-1}\right)$ and $C=-\lambda_{1}^{2} e_{1} \wedge e_{2}+\lambda_{1}\left(\lambda_{1}^{2}-1\right)^{\frac{1}{2}} e_{2} \wedge e_{4}$.

Theorem 3.7. A non- minimal rotational surfaces of hyperbolic type $M_{2}$ defined by (3.33) has pointwise 1-type Gauss map of the first kind if and only if $M_{2}$ has parallel mean curvature vector

Corollary 3.8. If rotational surfaces of hyperbolic type $M_{2}$ given by (3.33) is minimal then it has pointwise 1-type Gauss map of the first kind.

\subsection{Rotational surfaces of parabolic type with pointwise 1-type Gauss map in $\mathbb{E}_{2}^{4}$}

In this subsection, we study rotational surfaces of parabolic type with pointwise 1-type Gauss map. We show that flat rotational surface of parabolic type has pointwise 1-type Gauss map if and only if its Gauss map is harmonic. Also we conclude that flat rotational surface of parabolic type has harmonic Gauss map if and only if it has parallel mean curvature vector. 
We consider the pseudo-orthonormal base $\left\{\epsilon_{1}, \xi_{2}, \xi_{3}, \epsilon_{4}\right\}$ of $\mathbb{E}_{2}^{4}$ such that $\xi_{2}=$ $\frac{\epsilon_{2}+\epsilon_{3}}{\sqrt{2}}, \xi_{3}=\frac{-\epsilon_{2}+\epsilon_{3}}{\sqrt{2}}\left\langle\xi_{2}, \xi_{2}\right\rangle=\left\langle\xi_{3}, \xi_{3}\right\rangle=0$ and $\left\langle\xi_{2}, \xi_{3}\right\rangle=-1$. Let consider $\alpha$ spacelike curve is given by

$$
x(s)=x_{1}(s) \epsilon_{1}+x_{2}(s) \epsilon_{2}+x_{3}(s) \epsilon_{3}
$$

or we can express $x$ according to pseudo-orthonormal base $\left\{\epsilon_{1}, \xi_{2}, \xi_{3}, \epsilon_{4}\right\}$ as follows:

$$
x(s)=x_{1}(s) \epsilon_{1}+p(s) \xi_{2}+q(s) \xi_{3},
$$

where $p(s)=\frac{x_{2}(s)+x_{3}(s)}{\sqrt{2}}$ and $q(s)=\frac{-x_{2}(s)+x_{3}(s)}{\sqrt{2}}$. The rotational surface of parabolic type $M_{3}$ is defined by

$$
M_{3}: \varphi(t, s)=x_{1}(s) \epsilon_{1}+p(s) \xi_{2}+\left(-t^{2} p(s)+q(s)\right) \xi_{3}+\sqrt{2} t p(s) \epsilon_{4},
$$

We suppose that $x$ is parameterized by arc-length, that is, $\left(x_{1}{ }^{\prime}(s)\right)^{2}-2 p^{\prime}(s) q^{\prime}(s)=$ 1 . Now we can give a moving orthonormal frame $\left\{e_{1}, e_{2}, e_{3}, e_{4}\right\}$ for $M_{3}$ as follows:

$$
\begin{aligned}
& e_{1}=x_{1}{ }^{\prime}(s) \epsilon_{1}+p^{\prime}(s) \xi_{2}+\left(-t^{2} p^{\prime}(s)+q^{\prime}(s)\right) \xi_{3}+\sqrt{2} t p^{\prime}(s) \epsilon_{4}, \\
& e_{2}=-\sqrt{2} t \xi_{3}+\epsilon_{4}, \\
& e_{3}=\epsilon_{1}+\frac{x_{1}{ }^{\prime}(s)}{p^{\prime}(s)} \xi_{3}, \\
& e_{4}=x_{1}{ }^{\prime}(s) \epsilon_{1}+p^{\prime}(s) \xi_{2}+\left(\frac{1}{p^{\prime}(s)}+q^{\prime}(s)-t^{2} p^{\prime}(s)\right) \xi_{3}+\sqrt{2} t p^{\prime}(s) \epsilon_{4},
\end{aligned}
$$

where $p^{\prime}(s)$ is non zero. Then it is easily seen that

$$
\left\langle e_{1}, e_{1}\right\rangle=\left\langle e_{3}, e_{3}\right\rangle=1, \quad\left\langle e_{2}, e_{2}\right\rangle=\left\langle e_{4}, e_{4}\right\rangle=-1 .
$$

We have the dual 1-forms as:

$$
\omega_{1}=d s \quad \text { and } \quad \omega_{2}=-\sqrt{2} p(s) d t .
$$

Also we obtain components of the second fundamental form and the connection forms as:

$$
\begin{aligned}
& h_{11}^{3}=c(s), h_{12}^{3}=0, h_{22}^{3}=0, \\
& h_{11}^{4}=-b(s), \quad h_{12}^{4}=0, \quad h_{22}^{4}=a(s)
\end{aligned}
$$

and

$$
\begin{aligned}
& \omega_{12}=a(s) \omega_{2}, \quad \omega_{13}=c(s) \omega_{1}, \quad \omega_{14}=-b(s) \omega_{1}, \\
& \omega_{23}=0, \quad \omega_{24}=-a(s) \omega_{2}, \quad \omega_{34}=-c(s) \omega_{1} .
\end{aligned}
$$

Then, by taking the covariant derivatives with respect to $e_{1}$ and $e_{2}$, weget as 
follows:

$$
\begin{aligned}
\tilde{\nabla}_{e_{1}} e_{1} & =c(s) e_{3}+b(s) e_{4}, \\
\tilde{\nabla}_{e_{2}} e_{1} & =a(s) e_{2}, \\
\tilde{\nabla}_{e_{1}} e_{2} & =0, \\
\tilde{\nabla}_{e_{2}} e_{2} & =a(s) e_{1}-a(s) e_{4}, \\
\tilde{\nabla}_{e_{1}} e_{3} & =-c(s) e_{1}+c(s) e_{4}, \\
\tilde{\nabla}_{e_{2}} e_{3} & =0, \\
\tilde{\nabla}_{e_{1}} e_{4} & =b(s) e_{1}+c(s) e_{3}, \\
\tilde{\nabla}_{e_{2}} e_{4} & =a(s) e_{2},
\end{aligned}
$$

where

$$
\begin{gathered}
a(s)=\frac{p^{\prime}(s)}{p(s)}, \\
b(s)=\frac{p^{\prime \prime}(s)}{p^{\prime}(s)}, \\
c(s)=\frac{x_{1}^{\prime \prime}(s) p^{\prime}(s)-p^{\prime \prime}(s) x_{1}^{\prime}(s)}{p^{\prime}(s)} .
\end{gathered}
$$

By using (2.2), (2.3) and (3.37), the mean curvature vector and Gaussian curvature of the surface $M_{3}$ are obtained as follows:

$$
H=\frac{1}{2}\left(c(s) e_{3}+(a(s)+b(s)) e_{4}\right)
$$

and

$$
K=a(s) b(s),
$$

respectively.

By using (2.1) and (3.38), we find the Laplacian of the Gauss map of $M_{3}$ by

$$
\Delta G=L(s)\left(e_{1} \wedge e_{2}\right)+M(s)\left(e_{2} \wedge e_{3}\right)+N(s)\left(e_{2} \wedge e_{4}\right),
$$

where

$$
\begin{gathered}
L(s)=c^{2}(s)-a^{2}(s)-b^{2}(s), \\
M(s)=c^{\prime}(s)+c(s)(a(s)+b(s)), \\
N(s)=c^{2}(s)+a^{\prime}(s)+b^{\prime}(s) .
\end{gathered}
$$

Theorem 3.9. Let $M_{3}$ be flat rotation surface of parabolic type given by the parameterization (3.36). Then $M_{3}$ has pointwise 1-type Gauss map if and only if the profile curve of $M_{3}$ is given by

$$
\begin{aligned}
x_{1}(s) & =\frac{\varepsilon}{\mu_{1}}\left(\ln \left(\mu_{1} s+\mu_{2}\right)\left(\mu_{1} s+\mu_{2}\right)\right)+\left(\mu_{4}-\varepsilon\right) s+\mu_{5}, \\
p(s) & =\mu_{1} s+\mu_{2}, \\
q(s) & =\frac{1}{2 \mu_{1}} \int\left(\left(\varepsilon \ln \left(\mu_{1} s+\mu_{2}\right)+\mu_{4}\right)^{2}-1\right) d s,
\end{aligned}
$$


where $\mu_{1}, \mu_{2}, \mu_{4}, \mu_{5}$ real constants. Morever the surface $M_{3}$ has harmonic Gauss map for $f=0$.

Proof. We suppose that $M_{3}$ has pointwise 1-type Gauss map. In that case the Gauss map of $M_{3}$ satisfies (1.1). By using (1.1) and (3.44), we get

$$
\begin{aligned}
-f+f\left\langle C, e_{1} \wedge e_{2}\right\rangle & =-L(s), \\
f\left\langle C, e_{2} \wedge e_{3}\right\rangle & =-M(s), \\
f\left\langle C, e_{2} \wedge e_{4}\right\rangle & =N(s)
\end{aligned}
$$

and

$$
\left\langle C, e_{1} \wedge e_{3}\right\rangle=\left\langle C, e_{1} \wedge e_{4}\right\rangle=\left\langle C, e_{3} \wedge e_{4}\right\rangle=0 .
$$

By taking the derivatives of all equations in (3.49) with respect to $e_{2}$ and using (3.48) we obtain

$$
\begin{aligned}
L(s)-N(s) & =f, \\
M(s) & =0,
\end{aligned}
$$

respectively. Since the surface $M_{3}$ is flat, i.e., $K=0$ from (3.43) we have that $b(s)=0$. From (3.40) we obtain that

$$
p(s)=\mu_{1} s+\mu_{2}
$$

for some constants $\mu_{1} \neq 0$ and $\mu_{2}$. By using (3.39) and (3.51) we have that

$$
a(s)=\frac{\mu_{1}}{\mu_{1} s+\mu_{2}} .
$$

If we consider $M(s)=0$ with the equations $b(s)=0$ and $a(s)=\frac{\mu_{1}}{\mu_{1} s+\mu_{2}}$, from (3.46) we get

$$
c(s)=\frac{\mu_{3}}{\mu_{1} s+\mu_{2}} .
$$

On the other hand, by using the first equation of (3.50), (3.45), (3.47), (3.52) and (3.53) we obtain that $f=0$. It means that $L(s)=N(s)=0$ and we have

$$
\mu_{3}=\varepsilon \mu_{1}, \varepsilon= \pm 1 \text {. }
$$

If we consider $(3.41),(3.51)$ and (3.53) we get

$$
x_{1}(s)=\frac{\varepsilon}{\mu_{1}}\left(\ln \left(\mu_{1} s+\mu_{2}\right)\left(\mu_{1} s+\mu_{2}\right)\right)+\left(\mu_{4}-\varepsilon\right) s+\mu_{5},
$$

where $\mu_{4}, \mu_{5}$ are constants of integration. Since $x$ is unit speed spacelike curve we get

$$
q^{\prime}(s)=\frac{\left(x_{1}{ }^{\prime}(s)\right)^{2}-1}{2 p^{\prime}(s)} .
$$

By substituting (3.51) and (3.54) into (3.55) we obtain

$$
q(s)=\frac{1}{2 \mu_{1}} \int\left(\left(\varepsilon \ln \left(\mu_{1} s+\mu_{2}\right)+\mu_{4}\right)^{2}-1\right) d s .
$$

This completes the proof. 
Theorem 3.10. Let $M_{3}$ be flat rotational surfaces of parabolic type given by (3.36). $M_{3}$ has harmonic Gauss map if and only if its mean curvature vector is parallel.

Proof. We suppose that $M_{3}$ has parallel mean curvature vector, i.e., $D H=0$. From (3.42) we have that

$$
D_{e_{1}} H=\frac{1}{2}\left(M(s) e_{3}+N(s) e_{4}\right)=0 .
$$

In this case we obtain that $M(s)=N(s)=0$. Since $M_{3}$ is a flat surface, from the previous theorem we have

$$
b(s)=0 \text { and } a(s)=\frac{\mu_{1}}{\mu_{1} s+\mu_{2}} .
$$

By considering the equation $M(s)=0$ with above equations and using (3.46) we get

$$
c(s)=\frac{\mu_{3}}{\mu_{1} s+\mu_{2}},
$$

where $\mu_{3}$ is the constant of integration. It implies that $L(s)=0$. Hence we obtain that Gauss map of $M_{3}$ is harmonic .

Conversely, if $M_{3}$ is harmonic then it is easily seen that $D H=0$.

The first author is supported by Ahi Evran University :PYO-EGF.4001.15.002.

\section{REFERENCES}

[1] Aksoyak, K. F., Yayl,, Y., General Rotational Surfaces with pointwise 1type Gauss map in pseudo- Euclidean space $\mathbb{E}_{2}^{4}$, Indian J. Pure Appl. Math., 46 (2015), 107-118.

[2] Aksoyak, K. F., Yayl,, Y., Boost invariant surfaces with pointwise 1-type Gauss map in Minkowski 4-space $\mathbb{E}_{1}^{4}$, Bull. Korean Math. Soc. 51 (2014), $1863-1874$.

[3] Arslan, K., Bayram, B. K., Bulca, B., Kim, Y. H., Murathan, C., Öztürk, G., Rotational embeddings in $\mathbb{E}_{1}^{4}$ with pointwise 1-type Gauss map, Turk. J. Math. 35 (2011), 493-499.

[4] Arslan, K., Bayram, B. K., Bulca, B., Kim, Y. H., Murathan, C., Öztürk, G., Vranceanu surface in $E^{4}$ with pointwise 1-type Gauss map, Indian J. Pure. Appl. Math. 42 (2011), 41-51.

[5] Bektaş, B., Dursun, U., Timelike rotational surfaces of elliptic, hyperbolic and parabolic types in Minkowski space $\mathbb{E}_{1}^{4}$ with pointwise 1-type Gauss map, Filomat 29 (2015), 381-392.

[6] Chen, B.-Y., Piccinni, P. Submanifolds with Finite Type-Gauss map, Bull. Austral. Math. Soc. 35 (1987), 161-186. 
[7] Dursun, U., Bektaş, B., Spacelike rotational rurfaces of elliptic, hyperbolic and parabolic types in Minkowski space $\mathbb{E}_{1}^{4}$ with pointwise 1-type Gauss map, Math. Phys. Anal. Geom. 17 (2014), 247-263.

[8] Dursun, U., On rotational spacelike surfaces in Minkowski space $\mathbb{E}_{1}^{4}$ with pointwise 1-type Gauss map, Bull. Korean Math. Soc. 52 (2015), 301-312.

[9] Dursun, U., Turgay, N. C., General rotational surfaces in Euclidean space $E^{4}$ with pointwise 1-type Gauss map, Math. Commun. 17 (2012), 71-81.

[10] Ganchev, G., Milousheva, V., Quasi-minimal rotational surfaces in pseudoEuclidean four-dimensional space, Cent. Eur. J. Math. 12 (2014), 15861601.

[11] Kim, Y. H., Yoon, D. W. Classification of rotation surfaces in pseudo Euclidean space, J. Korean Math. 41 (2004), 379-396. 